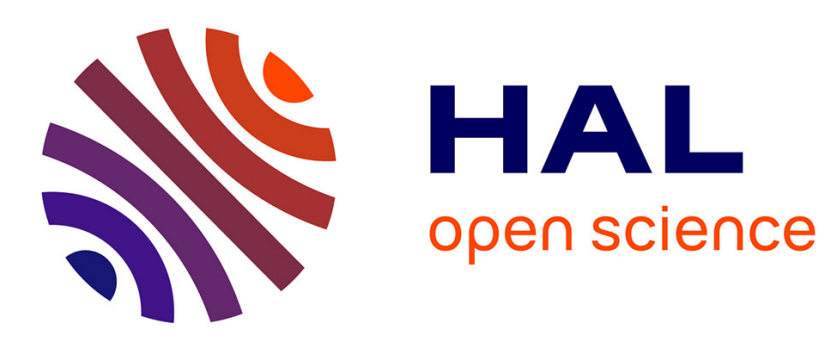

\title{
Flux estimation algorithms for electric drives: a comparative study
}

\author{
Mohamad Koteich
}

\section{To cite this version:}

Mohamad Koteich. Flux estimation algorithms for electric drives: a comparative study. International Conference on Renewable Energies for Developing countries (REDEC 2016), Jul 2016, Zouk Mosbeh, Lebanon. hal-01322795

\section{HAL Id: hal-01322795 \\ https://hal.science/hal-01322795}

Submitted on 31 May 2016

HAL is a multi-disciplinary open access archive for the deposit and dissemination of scientific research documents, whether they are published or not. The documents may come from teaching and research institutions in France or abroad, or from public or private research centers.
L'archive ouverte pluridisciplinaire HAL, est destinée au dépôt et à la diffusion de documents scientifiques de niveau recherche, publiés ou non, émanant des établissements d'enseignement et de recherche français ou étrangers, des laboratoires publics ou privés. 


\title{
Flux estimation algorithms for electric drives: a comparative study
}

\author{
Mohamad Koteich \\ Renault Group, Technocentre \\ 1 Avenue du Golf, 78288 Guyancourt, France \\ mohamad.koteich@renault.com
}

\begin{abstract}
This paper reviews the stator flux estimation algorithms applied to the alternating current motor drives. The socalled voltage model estimation, which consists of integrating the back-electromotive force signal, is addressed. However, in practice, the pure integration is prone to drift problems due to noises, measurement error, stator resistance uncertainty and unknown initial conditions. This limitation becomes more restrictive at low speed operation. Several solutions, reported in the literature, are reviewed and compared. Emphasis is placed on the low-pass filter based algorithms that show good performance in steady-state as well as in transient operating conditions.
\end{abstract}

\section{INTRODUCTION}

High-performance motor drives, such as field-oriented controlled and direct-torque controlled alternating current (AC) drives, require an accurate stator flux estimation. For $\mathrm{AC}$ machines, there exist two models for flux estimation, namely the voltage model and the current model.

The current model estimation is known to be efficient in low and medium speed range, especially when combined with high-frequency injection-based estimation techniques [1]. Nevertheless, the accuracy of the current model is highly dependent on the knowledge of the machine inductances. On the other hand, the voltage model, which consists of integrating the stator back-electromotive force (EMF) signal, is known for its good performance at medium and high speeds. The main advantage of the voltage model is its robustness against the machine parameters; it only requires the stator resistance, which can be quite accurately known in various applications.

Both voltage and current models can be combined in one state-observer estimation algorithm [2], [3], [4], [5]. Note that the voltage model implementation is the same for all $\mathrm{AC}$ drives, whereas the current model depends on the machine structure. In this paper, the voltage model is addressed.

The implementation of a pure integrator is prone to drift problems due to the following practical issues [6]: a) Inverter Nonlinearity: the stator voltages are not directly measured, they are constructed using the reference voltage of the pulsewidth modulator (PWM). This provides a 'clean' voltage signal, but it does not exactly represent the stator voltages as the PWM inverter introduces distortions. b) Current Measurement: the current measurement channels exhibits errors due to unbalanced gains and DC drift. c) Stator Resistance: the accurate knowledge of the stator resistance, which may vary, is important for accurate estimation. d) Integrator initial conditions: unknown initial conditions, at the starting of the drive or when severe back-EMF changes occur, result in a DC-offset in the integrator output.
Various algorithms have been reported in the literature to solve the drift problems. One simple solution consists of using a slight amount of low-pass filtering in the integration of the back-EMF [7]. However, this introduces errors in the estimated flux signal especially when the motor frequency is lower than the cutoff frequency of the low-pass filter (LPF) [8]. In view of emulating the frequency response of a pure integrator, the authors of [9] propose a programmable cascaded LPF method of flux estimation: three cascaded programmable LPFs with magnitude compensation are designed and shown to be efficient when applied for sensorless stator-flux-oriented control of induction machines. In the same context, several compensated LPF algorithms have been proposed for directtorque controlled [10] and field-oriented controlled [8], [11], [12] AC machines. Other algorithms, concentrated on the DCoffset rejection, consist of limiting the amplitude of the output flux signal using an adequate saturation function [13], [14], [15].

This paper is focused on the estimation of the stator flux of $\mathrm{AC}$ machines using the voltage model. It reviews the estimation algorithms reported in the literature, and summarizes them in one generalized estimation algorithm, as detailed in Section II. From the generalized algorithm, three categories of modified integrators can be derived: 1) low-pass filter (LPF) based algorithms, which are seen as open-loop estimators, 2) amplitude saturation integrators and 3) adaptive flux observers. The last two categories are considered to be closed-loop estimators since the estimated flux is compared to a certain correction signal in a feedback structure. Section III is dedicated for the study of LPF-based algorithms with a detailed comparative study using digital simulation. Section IV reviews the correction-based estimation algorithms. Conclusions are drawn in Section V.

\section{StATOR FLUX ESTIMATION PROBLEM}

A stator winding of an electric motor can be seen as a connection of a resistance $R_{s}$ in series with a coil having time-varying inductance. Throughout this paper, the complex space-vector notation is used to represent the electromagnetic quantities (the currents $\underline{i}_{s}$, voltages $\underline{v}_{s}$ and fluxes $\underline{\psi}_{s}$ ). The voltage model equation can be written as:

$$
\underline{v}_{s}=R_{s} \underline{i}_{s}+\frac{d \underline{\psi}_{s}}{d t}
$$

Therefore, the stator flux vector can be estimated by integrating the back-EMF $\left(\underline{e}_{s}\right)$ :

$$
\underline{\psi}_{s}=\int\left(\underline{v}_{s}-R_{s} \underline{i}_{s}\right)=\int \underline{e}_{s}
$$


The frequency response of the integrator in the Laplace domain is:

$$
\frac{\Psi_{s}(s)}{\mathbf{e}_{s}(s)}=\frac{1}{s}
$$

The magnitude and the phase lag of the integrator are:

$$
\begin{aligned}
G & =\frac{1}{\left|\omega_{s}\right|} \\
\varphi & =-\frac{\pi}{2}
\end{aligned}
$$

where $\omega_{s}$ is the angular frequency of the stator flux $\Psi_{s}(s)$.

To ensure an accurate estimation, several algorithms have been reported in the literature. They can be summarized using the following general formulation:

$$
\underline{\hat{\psi}}_{s}=\underline{e}_{s}+\omega_{c}(t)\left(\underline{\psi}_{s}^{c o r}-\underline{\psi}_{s}\right)
$$

This equation is illustrated graphically on Fig. 1. It is written similarly to the traditional state-observer equation:

$$
\dot{\hat{x}}=f(x, u)+\omega_{c}(t)(y-\hat{y})
$$

where $x$ is the state, $u$ is the input, $y$ is the output, $f$ is the dynamical model of the system and $\omega_{c}(t)$ is the observer gain to be tuned. However, the equation (6) is not an exact stateobserver since $y$ is not a measured signal; a correction flux, $\underline{\psi}_{s}^{c o r}$, is used for comparison with the estimated flux.

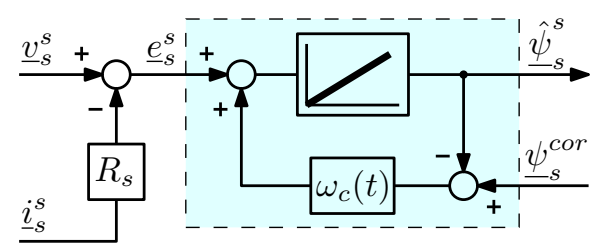

Fig. 1: Generalized modified integrator structure

In the frequency domain, the equation (6) becomes ${ }^{1}$ :

$$
\underline{\Psi}_{s}=\frac{\underline{\mathbf{e}}_{s}+\omega_{c} \underline{\Psi}_{s}^{c o r}}{s+\omega_{c}}
$$

The generality of this structure lies in the choice of $\underline{\psi}_{s}^{\text {cor }}$; depending on this choice different estimation algorithms can be derived. At a first glance, two particular algorithms prevail. Choosing

$$
\underline{\psi}_{s}^{c o r}=\underline{\psi}_{s}
$$

yields the pure integrator equation, and choosing

$$
\underline{\psi}_{s}^{c o r}=0
$$

results in a low-pass filter equation, with a corner frequency $\omega_{c}$. The LPF algorithm is studied in details in the following section. Other possible choices of the term $\underline{\psi}_{s}^{\text {cor }}$ are discussed in section IV.

\footnotetext{
${ }^{1} \omega_{c}(t)$ is considered to be constant
}

\section{LOW-PASS FILTER}

One intuitive solution for DC-offset is to implement a highpass filter, with a corner frequency $\omega_{c}$, in series to the pure integrator. This results in the low-pass filter approximation of the integrator:

$$
\frac{\widehat{\Psi}_{s}}{\mathbf{e}_{s}}=\frac{1}{s} \cdot \frac{s}{s+\omega_{c}}=\frac{1}{s+\omega_{c}}
$$

A LPF can be seen as an integrator with a negative feedback, as shows the signal flow diagram of Fig. 2.

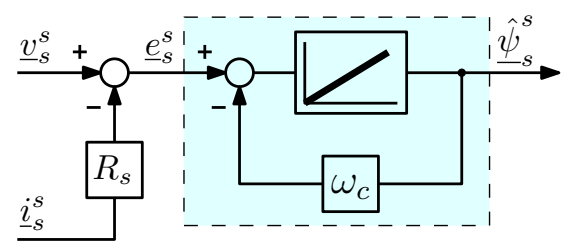

Fig. 2: Low-pass filter approximated integrator

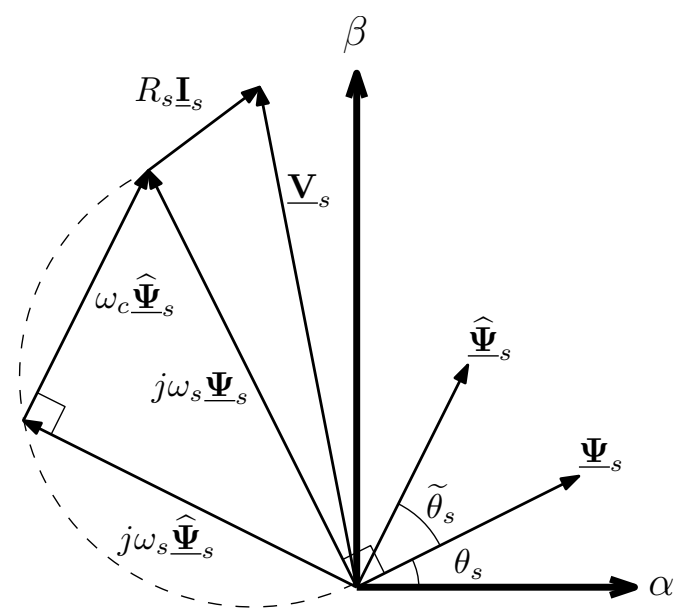

Fig. 3: Vector diagram of an LP

Under sinusoidal steady-state condition, the voltage model reduces to:

$$
\underline{\Psi}_{s}=\frac{1}{j \omega_{s}}\left(\underline{\mathbf{V}}_{s}-R_{s} \underline{\mathbf{I}}_{s}\right)
$$

whereas the LPF approximated integrator gives:

$$
\underline{\underline{\Psi}}_{s}=\frac{1}{j \omega_{s}+\omega_{c}}\left(\underline{\mathbf{V}}_{s}-R_{s} \underline{\mathbf{I}}_{s}\right)
$$

The vector diagram of Fig. 3 illustrates the relation between the real flux $\underline{\Psi}_{s}$ and the estimated flux $\widehat{\Psi}_{s}$. If the corner frequency $\omega_{c}$ is very low, the LPF is brought closer to the pure integrator.

In contrast to the pure integrator, the LPF is able to eliminate the DC-offset in the estimated flux. Fig. 4 shows the time-domain response of both the pure integrator and the LPF (with $\omega_{c}=2 \mathrm{rd} / \mathrm{s}$ ) in presence of an initial estimation error of $0.01 W b$. 
The magnitude and the phase of the LPF frequency-domain response are, respectively:

$$
\begin{aligned}
G & =\frac{1}{\sqrt{\omega_{s}^{2}+\omega_{c}^{2}}} \\
\varphi & =-\arctan \left(\frac{\omega_{s}}{\omega_{c}}\right)
\end{aligned}
$$

Hence, higher corner frequency $\omega_{c}$ ensures faster DC-offset rejection, however, it introduces higher distortions to the output signal due to increasing attenuation and phase lag. The most critical situation prevails when the stator frequency $\omega_{s}$ is lower than the corner frequency $\omega_{c}$. On the other hand, if $\omega_{c}$ is chosen to be very low, which corresponds to a large time-constant of the LPF, the drift problem persists.

To overcome such situations, a compensation of the LPF gain and phase can be considered in order to guaranty a pureintegrator-like overall frequency response. Furthermore, the LPF pole (situated at $-\omega_{c}$ ) should be placed far enough from the origin to solve the drift problem [8], [11].

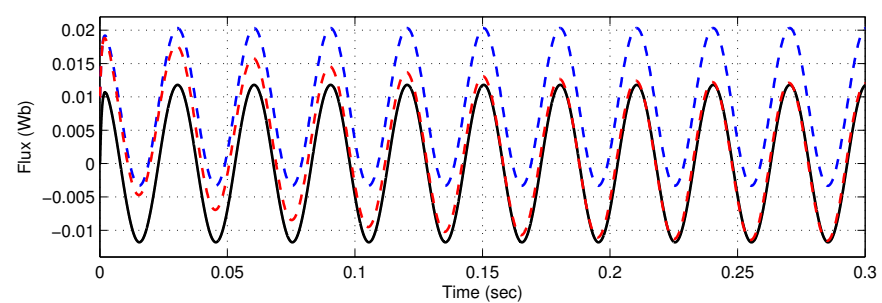

Fig. 4: Real flux (in black), integrator output (dashed blue) and low-pass filter output (dashed red) with $\omega_{c}=2 \mathrm{rd} / \mathrm{s}$.

\section{A. Compensation of the $L P F$}

Several solutions are proposed in the literature for LPF compensation [8], [10], [11], most of them are based on the multiplication of the LPF block by the following inverse of the HPF frequency response [11]:

$$
\frac{s+\omega_{c}}{s}=1+\frac{\omega_{c}}{s}
$$

Then, the compensation gain and phase lag are respectively:

$$
\begin{aligned}
G & =\frac{\sqrt{\omega_{s}^{2}+\omega_{c}^{2}}}{\left|\omega_{s}\right|} \\
\varphi & =\arctan \left(\frac{\omega_{s}}{\omega_{c}}\right)-\frac{\pi}{2}=-\arctan \left(\frac{\omega_{c}}{\omega_{s}}\right)
\end{aligned}
$$

Fig. 5 shows the frequency response of the pure integrator and two LPFs with cut-off frequencies of $1 \mathrm{rd} / \mathrm{s}$ and $10 \mathrm{rd} / \mathrm{s}$, as well as the frequency response of the corresponding compensation transfer function. The sum of an LPF response with its compensation response results in a pure integrator response.

This compensation can be applied to the output (flux) of the LPF [8], [10] as shown in Fig. 6a, or it can be applied to the back-emf signal, at the input of the LPF [11] as shown in Fig. 6b. Note that both compensation levels are not the same regarding the overall response, since it is not a simple multiplication of two linear time-invariant systems.

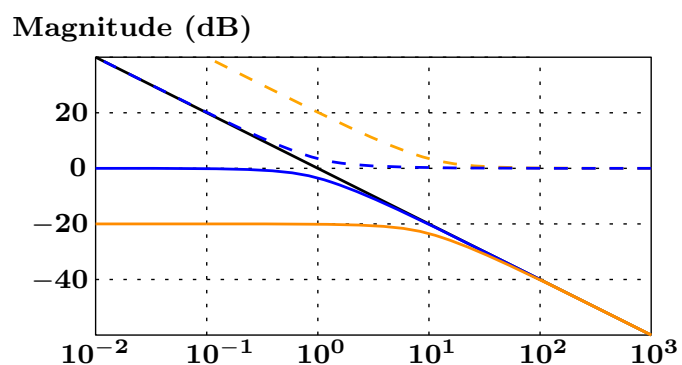

Phase (degree)

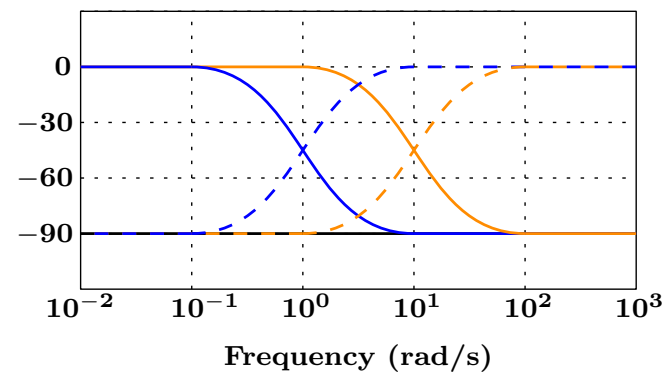

Fig. 5: Bode diagram of the pure integrator (black line) and the LPF for two cut-off frequencies $1 \mathrm{rd} / \mathrm{s}$ (blue line) and $10 \mathrm{rd} / \mathrm{s}$ (orange line), and the corresponding compensation transfer function (dashed lines).

Furthermore, for the same reason, the overall response depends on the corner frequency of the LPF/Compensation.

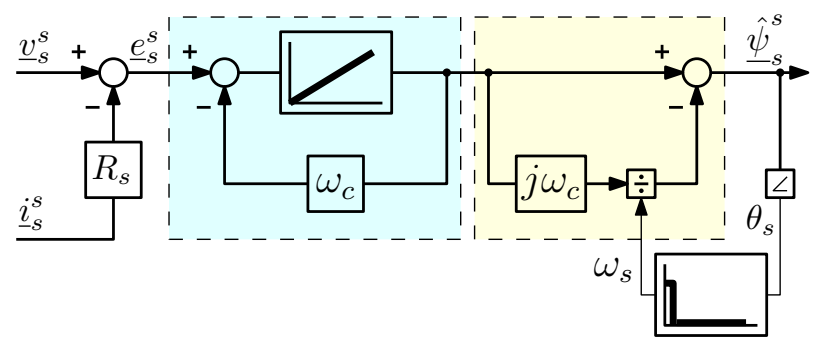

(a) output compensation [10]

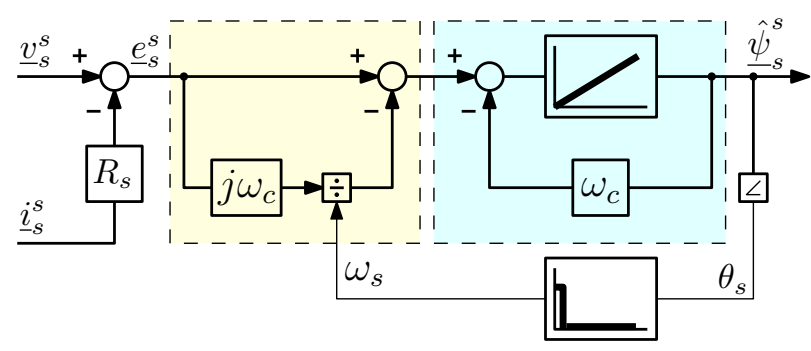

(b) input compensation

Fig. 6: Low-pass filter compensation

\section{B. Corner frequency tuning}

The choice of the the corner frequency is crucial for good flux estimation, especially in low-speed operating conditions: a filter that can rapidly attenuate the DC-offset in medium/high speed operation might fail at low-speed and vice-versa (see Fig. 7). This is due to the placement of the LPF pole with 
respect to the stator frequency. Hence, an adaptive corner frequency tuning can be adopted by choosing $\omega_{c}$ to be dependent on the stator angular frequency $\omega_{s}$ as follows:

$$
\omega_{c}=\lambda\left|\omega_{s}\right|
$$

where $\lambda$ is positive real number smaller than one. At low speed, $\lambda$ can be tuned to a low value, e.g. 0.1 , whereas for higher speeds, it can take higher values. In this case, the timeconstant of the LPF, $1 /\left(\lambda\left|\omega_{s}\right|\right)$, is decreased with the increase of the stator frequency.

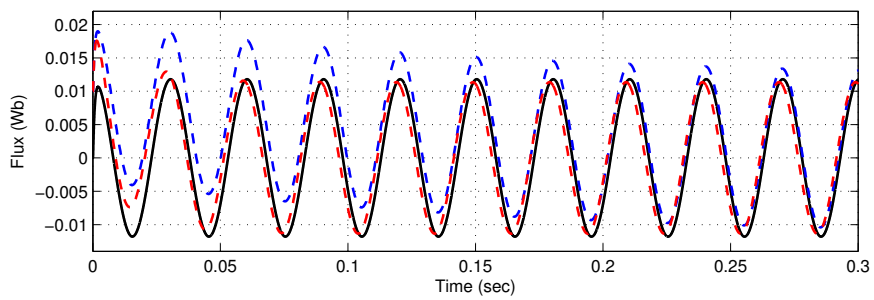

(a) Initial conditions error

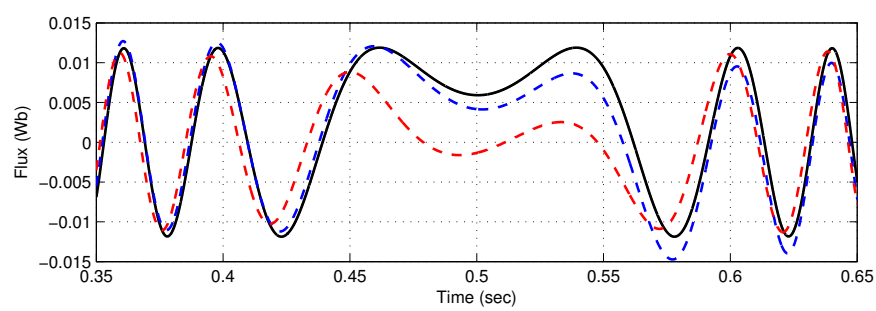

(b) Speed reversal

Fig. 7: Time response of the LPF for tow corner frequencies $\omega_{c}=1 \mathrm{rd} / \mathrm{s}$ (dashed blue) and $\omega_{c}=10 \mathrm{rd} / \mathrm{s}$ (dashed red).

There exist two ways to implement a compensated LPF with $\omega_{s}$-dependent corner frequency. The first one consists of multiplying the LPF output by the following gain $G$ and the phase lag $\varphi$ of the compensation function (Fig. 8a):

$$
\begin{aligned}
G & =\sqrt{1+\lambda^{2}} \\
\varphi & =-\operatorname{sign}\left(\omega_{s}\right) \arctan (\lambda)
\end{aligned}
$$

The other way is to apply the compensation to the back-EMF at the input of the LPF (Fig. 8b), which yields the following modified integrator equation [11]:

$$
\underline{\psi}_{s}=\int\left(-\lambda\left|\omega_{s}\right|+\left[1-j \lambda \operatorname{sign}\left(\omega_{s}\right)\right] \underline{e}_{s}\right) d t
$$

The comparison of the input-compensated and outputcompensated LPFs is shown in Fig. 9 for $\lambda=0.2$ : the input compensated LPF shows better behavior in the speed reversal operation, whereas the transient behavior in response to an initial estimation error seems to be the same for both estimators. Furthermore, the transient behavior is better if $\lambda$ is smaller, whereas a higher value of $\lambda$ allows faster rejection of the DC-offset (Fig. 10). Note that for $\lambda=0$ the pure integrator is achieved [11].

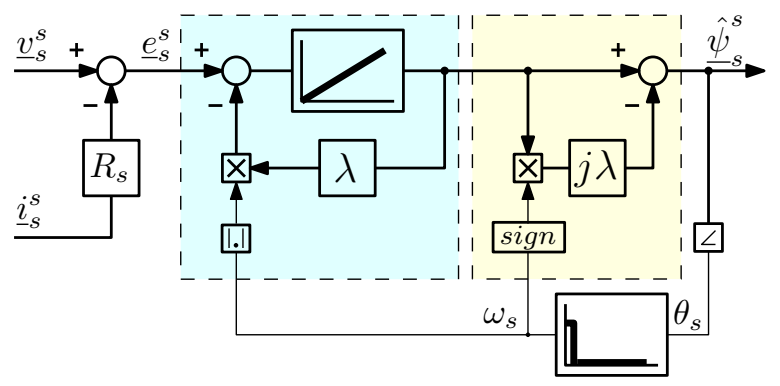

(a) Output compensation [8]

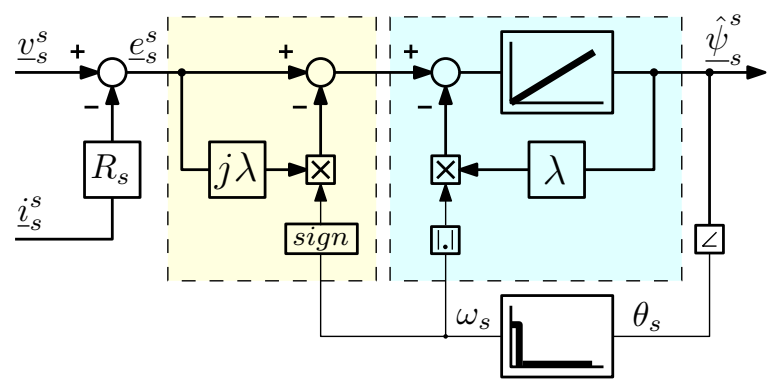

(b) Input compensation [11]

Fig. 8: Compensated low-pass filter with speed-dependent corner frequency $\omega_{c}=\lambda\left|\omega_{s}\right|$.

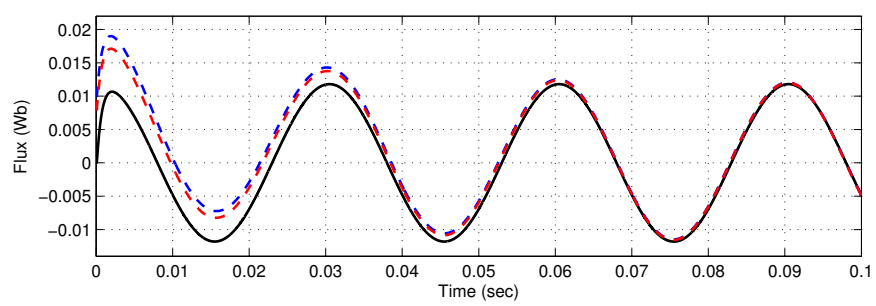

(a) Initial conditions error

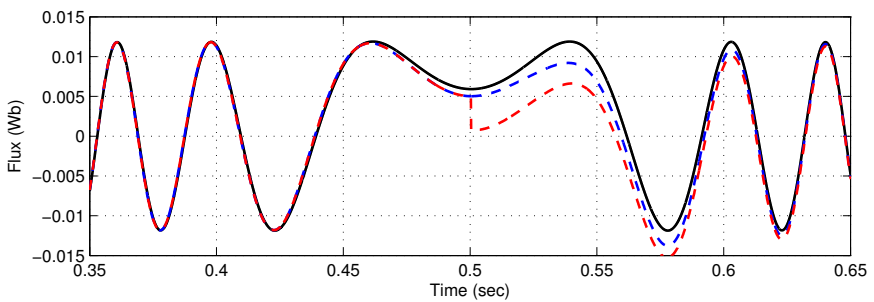

(b) Speed reversal

Fig. 9: Input compensated LPF (dashed blue) vs. output compensated LPF (dashed red) for $\lambda=0.2$.

\section{Stator frequency estimation}

In addition to the choice of $\lambda$, the performance of the compensated LPF depends heavily on the accuracy of the stator flux angular frequency $\left(\omega_{s}\right)$ estimate [16], which is often evaluated using the following equation:

$$
\omega_{s}=\frac{\left|\underline{e}_{s} \times \underline{\psi}_{s}\right|}{\left|\underline{\psi}_{s}\right|^{2}}=\frac{\psi_{s \alpha}\left(v_{s \beta}-R_{s} i_{s \beta}\right)-\psi_{s \beta}\left(v_{s \alpha}-R_{s} i_{s \alpha}\right)}{\psi_{s \alpha}^{2}+\psi_{s \beta}^{2}}
$$

However, this estimation is sensitive to the stator resistance uncertainty, to the offsets and distortions in the flux and back- 


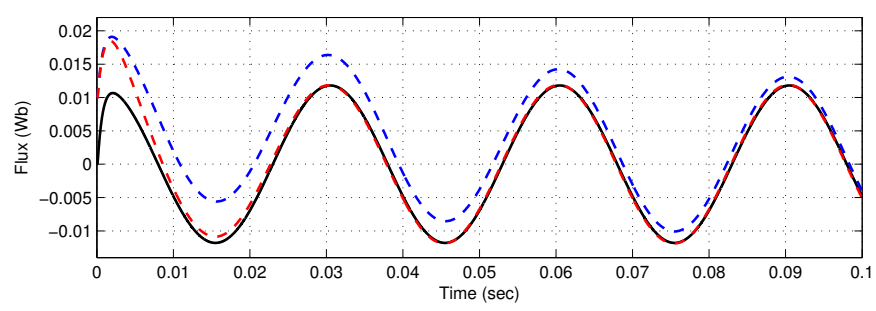

(a) Initial conditions error

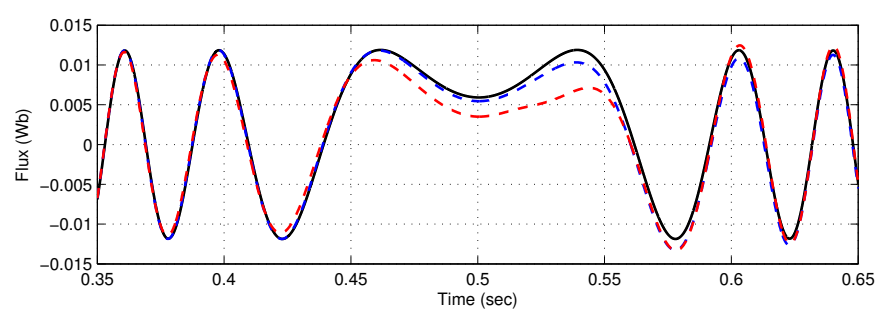

(b) Speed reversal

Fig. 10: Input compensated LPF with $\lambda=0.1$ (dashed blue) and $\lambda=0.9$ (dashed red).

EMF signals. In addition, the decreasing magnitude of the numerator at low speeds deteriorates the estimation. To remedy to this problem, the authors of [16] propose the PLL-based estimation scheme illustrated on Fig. 11. The stator voltage is used as a reference vector for the PLL, the voltage angle $\theta_{v}$ is synthesized and used as the angle of a rotational reference frame where the $q$-axis component of the voltage vector $\left(v_{s q}\right)$ should be null. Therefore, $v_{s q}$ is used as the error signal of a Proportional-Integral (PI) controller that outputs the $\omega_{s}$ estimate.

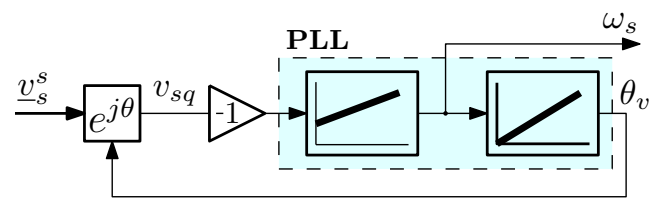

Fig. 11: PLL-based angular frequency estimation [16]

The choice of the voltage vector for the PLL is motivated by the fact that this vector has several advantages over the fluxes and current vectors: it is the very clean vector available, especially when constructed from the DC-link voltage and the switching states of the inverter, it has a considerable magnitude and generates a consistent large-enough error signal at the input of the PI [16].

\section{CORRECTION-BASED INTEGRATOR}

Although the LPF-based modified integrator provides a certain amount of output feedback, it is seen as an open-loop estimator since it corresponds to $\underline{\psi}_{s}^{\text {cor }}=0$ in the generalized modified integrator (6). This section presents another class of correction-based modified integrators, based on different choices of the correction flux $\psi_{s}^{\text {cor }}$. Two broad categories are presented: amplitude saturation integrator and adaptive flux observer.

\section{A. Amplitude saturation integrator}

The amplitude saturation integration algorithms consist of choosing a correction flux having the same phase of the integration output, but whose magnitude is seen as a saturation that limits the amplitude of the integrator output. A first, primitive, algorithm was proposed by [13] (Fig. 12a): the correction flux $\underline{\psi}_{s}^{c o r}$ is equal to the integrator output flux $\underline{\psi}_{S}$ (which yields a pure integrator) until the limiting level $L$ is exceeded. When $L$ is reached, the integrator output becomes:

$$
\underline{\hat{\Psi}}_{s}=\frac{\underline{\mathbf{e}}_{s}}{s+\omega_{c}}+\frac{\omega_{c}}{s+\omega_{c}} Z(L)
$$

where $Z(L)$ is the output of the saturation block whose amplitude is limited to $L$ [13]. One main limitation of the use of this algorithm is the tuning of $L$. Ideally, the limiting level $L$ should be equal to the actual flux amplitude in order to eliminate the DC-offset at the output. If $L$ is greater than the flux amplitude, the output waveform will have a DC component, in addition to the $\mathrm{AC}$ component, in response to an input offset. On the other hand, if $L$ is lower than the flux amplitude, the estimated flux waveform will be distorted.

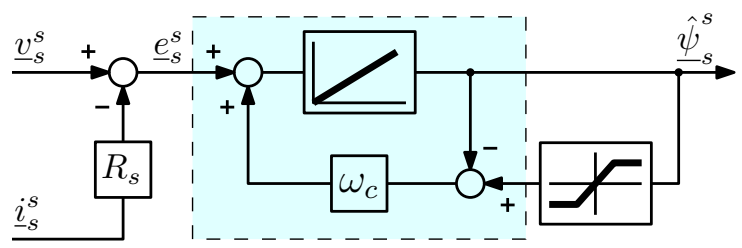

(a) Saturable feedback

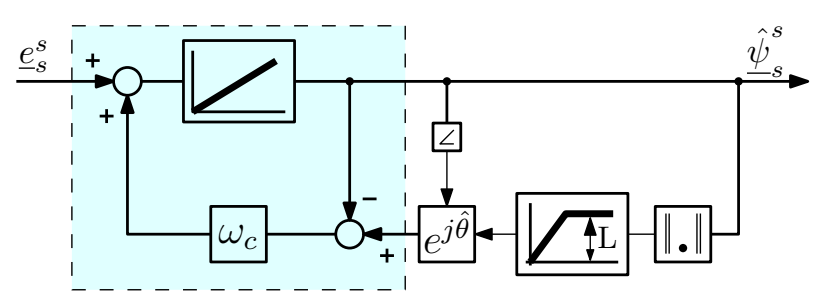

(b) Amplitude limiter

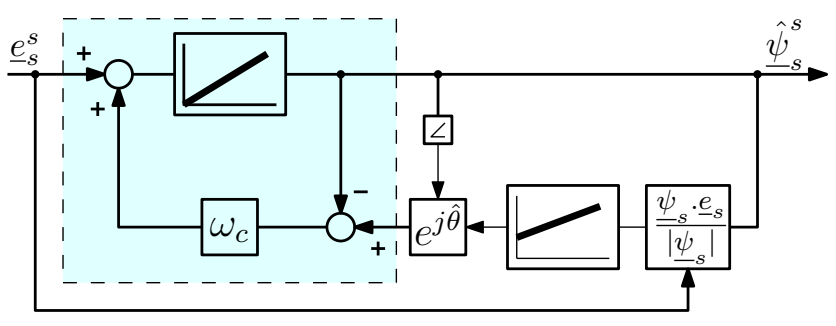

(c) Adaptive compensation using quadrature detector

Fig. 12: Modified integration algorithms proposed by [13]

Another algorithm, shown on the Fig. 12b was proposed in [13] to avoid possible waveform distortion when $L$ is lower than the actual flux amplitude: only the estimated flux amplitude is saturated to a limiting level $L$. Based on this algorithm, the authors of [13] design a third algorithm in which the value of $L$ is determined using an adaptive controller. The error signal fed to this controller is the dot product of the integrator's input (back-EMF) and output (estimated flux). 
This error is zero when the input and the output vectors are orthogonal (Fig. 3). Other papers, such as [14], [15] propose to take the amplitude of the stator flux reference (set-point) as a limiting level for the integrator, as shown on Fig. 13. Note that in [15] the corner frequency $\omega_{c}$ contains an integration term ( $\omega_{c}$ is time-variant) in order to have a PI controller fed with the difference between the estimated flux and the reference flux. However, the effectiveness of the integration action is not proved.

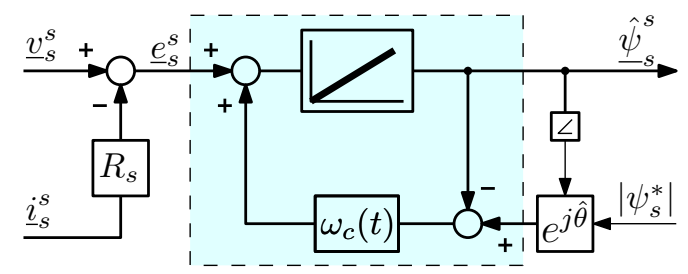

Fig. 13: Amplitude limitation modified integrator [14]

\section{B. Adaptive flux observers}

The adaptive flux observers are more complex estimation algorithms where the correction flux is the estimation of the stator flux using the current model [2], [3]. The general structure of the adaptive-observer-based flux estimator is shown on Fig. 14, where $L_{e q}=L_{q}$ for synchronous machines and $L_{e q}=L_{s}-M^{2} / L_{r}$ for induction machines [17].

In low-speed operating condition the performance of both voltage and current models is deteriorated. A high-frequency injection-based flux estimation can be applied to provide a more accurate current-model-based correction flux [1], [4], [18], [19].

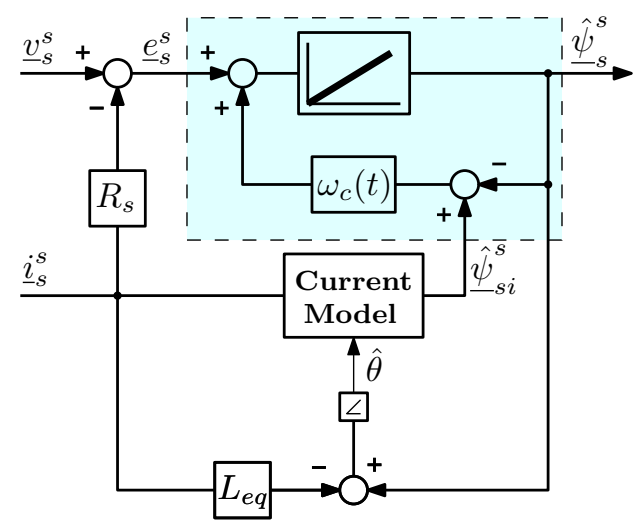

Fig. 14: Observer-based integrator

\section{CONCLUDING REMARKS}

A review of the stator flux estimation algorithms has been presented in this paper. As a conclusion, an input-compensated LPF with varying corner frequency can be a simple and efficient flux estimator. Further improvement of the reviewed algorithms may be realized by adapting (identifying) the stator resistance. This challenging topic has been addressed in the literature [14], [15], and still needs further investigations.

\section{REFERENCES}

[1] C. Silva, G. M. Asher, and M. Sumner, "Hybrid rotor position observer for wide speed-range sensorless pm motor drives including zero speed," IEEE Transactions on Industrial Electronics, vol. 53, no. 2, pp. 373-378, 2006.

[2] C. Lascu, I. Boldea, and F. Blaabjerg, "A modified direct torque control for induction motor sensorless drive," IEEE Transactions on Industry Applications, vol. 36, no. 1, pp. 122-130, 2000.

[3] I. Boldea, M. C. Paicu, and G.-D. Andreescu, "Active flux concept for motion-sensorless unified ac drives," IEEE Transactions on Power Electronics, vol. 23, pp. 2612-2618, 92008.

[4] F. J. W. Barnard, W. T. Villet, and M. J. Kamper, "Hybrid activeflux and arbitrary injection position sensorless control of reluctance synchronous machines," IEEE Transactions on Industry Applications, vol. 51, pp. 3899-3906, 92015.

[5] G. Foo and M. F. Rahman, "Sensorless direct torque and flux-controlled ipm synchronous motor drive at very low speed without signal injection," IEEE Transactions on Industrial Electronics, vol. 57, no. 1, pp. 395-403, 2010.

[6] J. Holtz and J. Quan, "Drift- and parameter-compensated flux estimator for persistent zero-stator-frequency operation of sensorless-controlled induction motors," IEEE Transactions on Industry Applications, vol. 39, pp. 1052-1060, 72003.

[7] K. D. Hurst, T. G. Habetler, G. Griva, and F. Profumo, "Zero-speed tacholess im torque control: simply a matter of stator voltage integration," IEEE Transactions on Industry Applications, vol. 34, no. 4, pp. 790-795, 1998.

[8] M.-h. Shin, D.-s. Hyun, S.-b. Cho, and S.-y. Choe, "An improved stator flux estimation for speed sensorless stator flux orientation control of induction motors," IEEE Transactions on Power Electronics, vol. 15, no. 2, pp. 312-318, 2000.

[9] B. Bose and N. Patel, "A programmable cascaded low-pass filter-based flux synthesis for a stator flux-oriented vector-controlled induction motor drive," IEEE Transactions on Industrial Electronics, vol. 44, no. 1, pp. 140-143, 1997.

[10] N. Idris and A. Yatim, "An improved stator flux estimation in steadystate operation for direct torque control of induction machines," IEEE Transactions on Industry Applications, vol. 38, no. 1, pp. 110-116, 2002.

[11] M. Hinkkanen and J. Luomi, "Modified integrator for voltage model flux estimation of induction motors," IEEE Transactions on Industrial Electronics, vol. 50, no. 4, pp. 818-820, 2003.

[12] D. Stojic, M. Milinkovic, S. Veinovic, and I. Klasnic, "Improved stator flux estimator for speed sensorless induction motor drives," IEEE Transactions on Power Electronics, vol. 30, pp. 2363-2371, 42015.

[13] J. Hu and B. Wu, "New integration algorithms for estimating motor flux over a wide speed range," IEEE Transactions on Power Electronics, vol. 13, no. 5, pp. 969-977, 1998.

[14] J. Holtz and J. Quan, "Sensorless vector control of induction motors at very low speed using a nonlinear inverter model and parameter identification," IEEE Transactions on Industry Applications, vol. 38, pp. 1087-1095, 72002.

[15] C. Lascu and G. D. Andreescu, "Sliding-mode observer and improved integrator with dc-offset compensation for flux estimation in sensorlesscontrolled induction motors," IEEE Transactions on Industrial Electronics, vol. 53, no. 3, pp. 785-794, 2006.

[16] M. Comanescu, L. Xu, and S. Member, "An improved flux observer based on pll frequency estimator for sensorless vector control of induction motors," Industrial Electronics, IEEE Transactions on, vol. 53, no. 1, pp. 50-56, 2005.

[17] M. Koteich, G. Duc, A. Maloum, and G. Sandou, "A unified model for low-cost high-performance ac drives: the equivalent flux concept," in The Third International Conference on Electrical, Electronics, Computer Engineering and their Applications (EECEA), Apr. 2016.

[18] G. D. Andreescu, C. I. Pitic, F. Blaabjerg, and I. Boldea, "Combined flux observer with signal injection enhancement for wide speed range sensorless direct torque control of ipmsm drives," IEEE Transactions on Energy Conversion, vol. 23, no. 2, pp. 393-402, 2008

[19] S.-c. Agarlita, I. Boldea, and F. Blaabjerg, "High-frequency-injectionassisted active-flux-based sensorless vector control of reluctance synchronous motors, with experiments from zero speed," IEEE Transactions on Industry Applications, vol. 48, pp. 1931-1939, 112012. 